\title{
Emergence of Complex Spatio-Temporal Order in Nonlinear Field Theories
}

\author{
Marcelo Gleiser \\ Department of Physics and Astronomy, Dartmouth College, Hanover, NH 03755, USA
}

\author{
Received on 01 August, 2006
}

\begin{abstract}
We investigate the emergence of time-dependent nonperturbative configurations during the evolution of nonlinear scalar field models with symmetric and asymmetric double-well potentials. Complex spatio-temporal behavior emerges as the system seeks to establish equipartition after a fast quench. We show that fast quenches may dramatically modify the decay rate of metastable states in first order phase transitions. We discuss possible applications in condensed matter systems and in inflationary cosmology.
\end{abstract}

Keywords: Phase transitions; Solitons; Symmetry breaking; Nucleation

\section{INTRODUCTION}

The emergence of complex patterns is one of the most distinct signatures of nonlinear interactions in natural systems. Since Einstein's pioneering work on Brownian motion [1], it became clear that much can be accomplished by modeling the interactions of a system with its environment through the action of random and viscous forces. During most of the twentieth century, studies were mainly restricted to investigating the motion of a point particle in nonlinear potentials [2]. With the advent of fast computers, modeling of stochastic evolution added spatial dimensions, allowing for the quantitative study of spatio-temporal complex behavior. Up to about ten years ago, most of the work concentrated in hydrodynamical and soft condensed-matter systems [3]. Recently, developments in high energy physics and cosmology have opened the interesting possibility that complex spatio-temporal behavior may also play a role in relativistic field theories, in particular during the early stages of cosmological evolution [4] and may even be observed in high-energy colliders [5].

Here we will briefly review some of the work done during the past few years which focused on understanding the effects of fast quenches on nonlinear scalar field theories. The quenches model both temperature quenches in the context of fast cosmological expansion (in particular at scales close to the GUT scale $\sim 10^{16} \mathrm{GeV}$ ) or the cooling of fireballs during high energy collisions such as those currently being investigated at RHIC and soon at LHC. The quench may also represent a pressure quench, common in condensed matter physics or, more generally, the appearance of a low-energy effective interaction that modifies the effective potential of the longwavelength modes of the field or order parameter describing the system's evolution. We will conclude with an application of these ideas to inflationary cosmology [6].

\section{THE MODEL}

Consider a $(2+1)$-dimensional real scalar field (or scalar order parameter) $\phi(\mathbf{x}, t)$ evolving under the influence of a potential $V(\phi)$. The continuum Hamiltonian is conserved and the total energy of a given field configuration $\phi(\mathbf{x}, t)$ is,

$$
H[\phi]=\int d^{2} x\left[\frac{1}{2}\left(\partial_{t} \phi\right)^{2}+\frac{1}{2}(\nabla \phi)^{2}+V(\phi)\right],
$$

where $V(\phi)=\frac{m^{2}}{2} \phi^{2}-\frac{\alpha}{3} \phi^{3}+\frac{\lambda}{8} \phi^{4}$ is the potential energy density. The parameters $m, \alpha$, and $\lambda$ are positive definite and temperature independent. It is helpful to introduce the dimensionless variables $\phi^{\prime}=\phi \sqrt{\lambda} / m, x^{\prime}=x m, t^{\prime}=t m$, and $\alpha^{\prime}=\alpha /(m \sqrt{\lambda})$ (We will henceforth drop the primes). Prior to the quench, $\alpha=0$ and the potential is an anharmonic single well symmetric about $\phi=0$. The field is in thermal equilibrium with a temperature $T$. At the temperatures considered, the fluctuations of the field are well approximated by a Gaussian distribution, with $\left\langle\phi^{2}\right\rangle=a T$ ( $a=0.51$ and can be computed numerically). As such, within the context of the Hartree approximation [7], the momentum and field modes in $k$-space can be obtained from a harmonic effective potential, and satisfy $\left\langle|\bar{\pi}(k)|^{2}\right\rangle=T$ and $\left\langle|\bar{\phi}(\mathbf{k})|^{2}\right\rangle=\frac{T}{k^{2}+m_{H}^{2}}$, respectively. The Hartree mass $m_{H}^{2}=1+\frac{3}{2}\left\langle\phi^{2}\right\rangle$ depends on the magnitude of the fluctuations (and thus $T$ ). Within the Hartree approximation we can write the effective potential as

$$
\begin{aligned}
V_{\mathrm{eff}}\left(\phi_{\mathrm{ave}}, m_{\mathrm{H}}^{2}\right)= & {\left[1-m_{\mathrm{H}}^{2}(t)\right] \phi_{\mathrm{ave}}+\frac{1}{2} m_{\mathrm{H}}^{2}(t) \phi_{\mathrm{ave}}^{2}-} \\
& -\frac{\alpha}{3} \phi_{\mathrm{ave}}^{3}+\frac{1}{8} \phi_{\mathrm{ave}}^{4} .
\end{aligned}
$$

Hereafter we will refer to a particular system by its initial temperature. All results are ensemble averages over 100 simulations.

If $\alpha \neq 0$, the $z_{2}$ symmetry is explicitly broken. When $\alpha=$ $1.5 \equiv \alpha_{c}$, the potential is a symmetric double-well (SDW), with two degenerate minima. This is the first case we consider.

\section{QUENCHING INTO SYMMETRIC DOUBLE WELLS: EMERGENCE OF SPATIO-TEMPORAL ORDER}

At $\alpha=\alpha_{c}=1.5$, the quench amounts to switching from a single to a double well with the field initially localized at $\phi=0$. In Fig. 1 we indicate this schematically.

As shown in Ref. [11], the quench sets oscillations in the field's zero mode, $\phi_{\text {ave }} \equiv \frac{1}{V} \int \phi d V$, where $V$ is the volume (or 


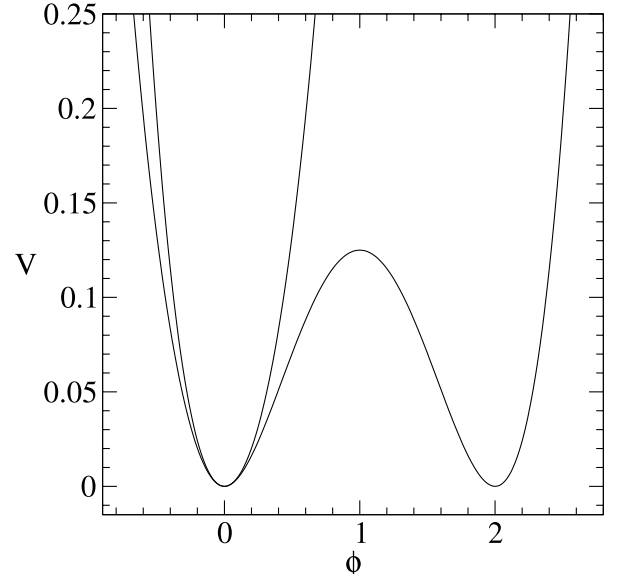

FIG. 1: Schematic picture showing change in potential $V(\phi)$ from single-well to symmetric double-well after quench.

area in 2d). The amplitude of these oscillations is controlled by the temperature of the initial Gaussian distribution, as explained above. In fact, temperature here is simply a convenient way to set an initial Gaussian distribution in momentum space. We did this using a Langevin equation with white noise. One could state that the field is at $T=0$ but initially set with a Gaussian distribution in momentum space with a certain width. This width is a measure of the initial "temperature" of the system.

At early times small fluctuations satisfy a Mathieu equation in $k$-space

$$
\ddot{\delta \phi}=-\left[k^{2}+V_{\mathrm{eff}}^{\prime \prime}\left[\phi_{\mathrm{ave}}(t)\right]\right] \delta \phi,
$$

and, depending on the wave number and parametric oscillations of $\phi_{\text {ave }}(t)$, can undergo exponential amplification $(\sim$ $\left.e^{\eta t}\right)$. For $T \leq 0.13$, no modes are ever amplified. As the temperature is increased, so is the amplitude and period of oscillation in $\phi_{\text {ave }}$, gradually causing the band $0<k<0.48$ to resonate and grow. Furthermore, for large enough temperatures $(T>0.13)$ large-amplitude fluctuations about the zero mode probe into unstable regions where $V_{\text {eff }}^{\prime \prime}<0$, which also promote their growth. Note that this is very distinct from spinodal decomposition, where competing domains of the two phases coarsen [8]. Instead, for the values of $T$ and $\alpha$ considered, $\phi_{\text {ave }}$ continues to oscillate about the $\phi=0$ minimum.

As a result of the energy transfer modeled by parametric amplification, oscillons are nucleated initially in phase. But what are oscillons? They are the higher dimensional equivalent of kink-antikink breathers, familiar of 1d nonlinear dynamics [9]. Extensive work has been done on oscillons and their properties and the reader can consult the relevant literature listed in Ref. [10]. Here, it is enough to mention that oscillons are long-lived, time-dependent, localized field configurations which express local ordering of momentum modes. What was also observed in Ref. [11] is that after the quench oscillons emerge in synchrony, exhibiting both spatial and time ordering. In Figure 2 we illustrate this phenomenon.

Finally, we introduce a measure of the partitioning of the kinetic energy $\Pi(t)$, which we use to describe the nonequilib-

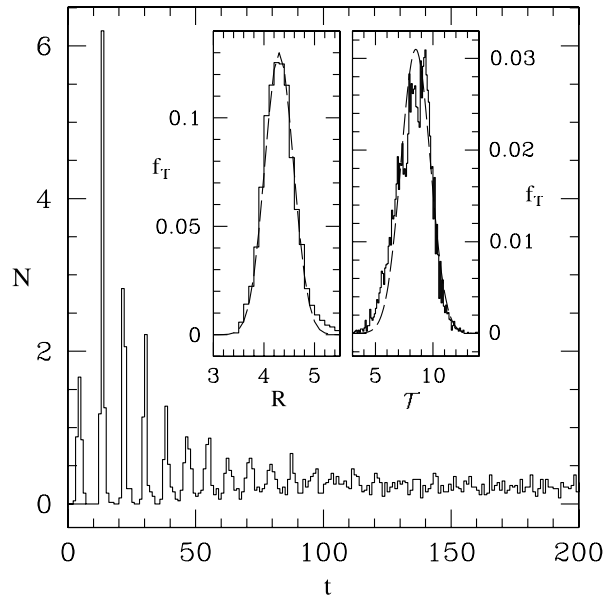

FIG. 2: Number of oscillons nucleated between $t$ and $t+\delta t$ at $T=0.22$ and $\delta t=1$. The global emergence is evident early in the simulations. Inset: probability distribution of radii and periods of oscillations of individual oscillons.

rium evolution of the system:

$$
\Pi(t)=-\int d^{2} k p(\mathbf{k}, t) \ln p(\mathbf{k}, t),
$$

where $p(\mathbf{k}, t)=K(\mathbf{k}, t) / \int d^{2} k K(\mathbf{k}, t)$, and $K(\mathbf{k}, t)$ is the kinetic energy of the $k$-th mode. $\Pi(t)$ attains its maximum $\left(\Pi_{\max }=\ln (N)\right.$ on a lattice with $N$ degrees of freedom) when equipartition is satisfied. This occurs both at the initial thermalization $(t=0)$ and final equilibrium states, since in this case all modes carry the same fractional kinetic energy. In Fig. 3 we show the change of $\Pi(t)$ from the initial state, $\Pi(t=0)-\Pi(t)$, for the closed system at $T=0.22$. At late times $(t>150)$, we have found that the system equilibrates exponentially in a time-scale $\tau \simeq 500$. At early times, the localization of energy at lower $\mathbf{k}$-modes, corresponding to the global emergence of oscillons, prolongs this approach to equipartition. The inset of Fig. 3 shows the large variations in $\Pi(t)$ (dotted line) that arise due to the synchronous oscillations in the kinetic energy of these configurations. Also shown (solid line) is the average between successive peaks of $\Pi(t)$, with a plateau at approximately $20<t<70$ that coincides with the maximum oscillon presence in the system. Thus, oscillon configurations serve as early bottlenecks to equipartition, temporarily suppressing the diffusion of energy from low $(0<|\mathbf{k}| \leq 0.8)$ to higher modes.

\section{QUENCHING INTO ASYMMETRIC DOUBLE WELLS: RESONANT NUCLEATION}

For $\alpha>\alpha_{c}=1.5$ the potential is asymmetric with the minimum at $\phi=0$ becoming metastable. We proceed as before by quenching the system from a single well, as illustrated in Fig. 4.

As before, oscillons will once again be nucleated. However, the situation now changes dramatically. Due to the asymmetry, the system will decay into the global minimum at $\phi_{+}$. 


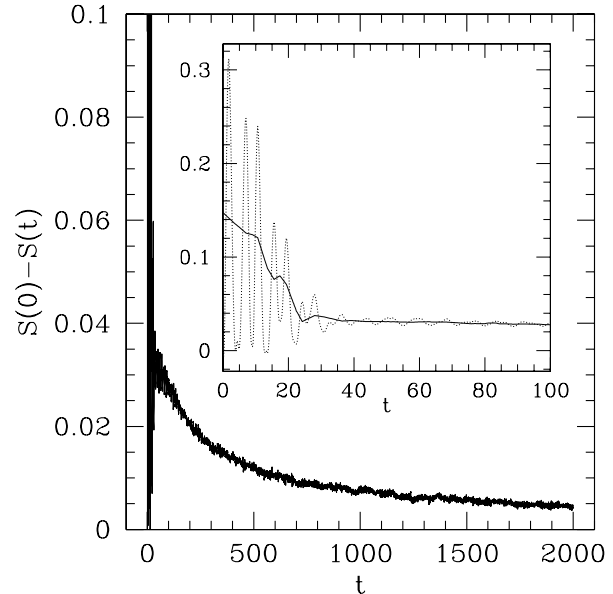

FIG. 3: The change of $\Pi(t)$ from the initial state for closed systems at $T=0.22$. The exponential approach to equilibrium is clear at late times. The inset illustrates the role of oscillons as a bottleneck to equipartition.

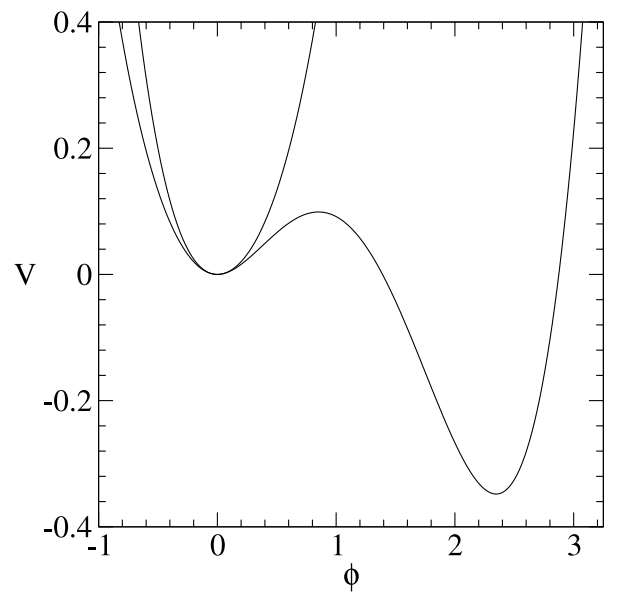

FIG. 4: Schematic picture showing change in potential $V(\phi)$ from single-well to asymmetric double-well after quench.

We have observed that this decay may occur in three possible ways depending on the initial temperature $T$ and the value of $\alpha$ [12]: i) the transition to the global minimum happens very fast in what can be called a "cross-over" transition; ii) the transition occurs as a single oscillon becomes unstable and grows into a critical bubble. As is well known from the theory of first order phase transitions [8], once a critical nucleus forms it will grow to complete the transition; iii) two or more oscillons percolate to become a critical bubble that then grows to complete the transition.

In order to simplify the analysis, we fixed the temperature to be $T \leq 0.22$. From the Hartree potential of Eq. 2, one can see that for large temperatures the potential becomes a single well again. For $T \leq 0.13$ no oscillons are nucleated after the quench. In this case, we expect that the usual metastable decay rate based on the theory of homogeneous nucleation (HN) will apply, becoming more accurate for smaller $T[8,13]$. The decay rate per unit volume obtained from

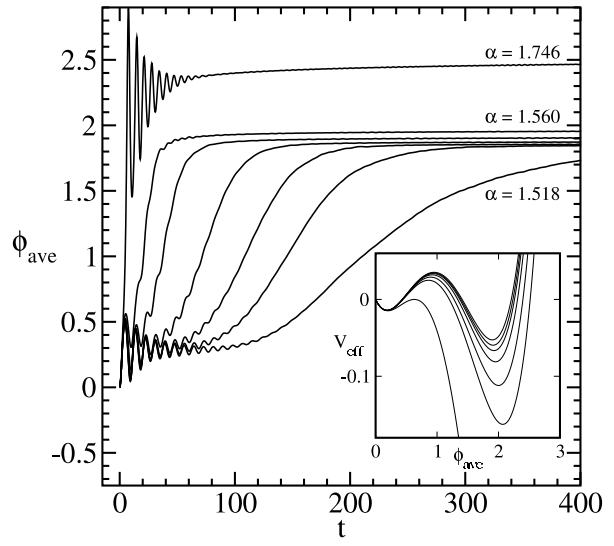

FIG. 5: The evolution of the order parameter $\phi_{\text {ave }}(t)$ at $T=0.22$ for several values of the asymmetry. From left to right, $\alpha=$ $1.746,1.56,1.542,1.53,1.524,1.521,1.518$. The inset shows $V_{\text {eff }}$ for the same values.

HN theory is controlled by the Arrhenius exponential suppression, $\Gamma(T, \alpha) \simeq T^{(d+1)} \exp \left[-E_{b}(T, \alpha) / T\right]$, where $E_{b}$ is the energy of the critical bubble or nucleus and $d$ is the number of spatial dimensions. [We use units where $c=k_{B}=\hbar=1$.] The typical time-scale for the decay in a volume $V$ is then, $\tau_{\mathrm{HN}} \simeq(V \Gamma)^{-1} \sim \exp \left[E_{b}(T, \alpha) / T\right]$.

In Fig. 5 we show the evolution of the order parameter $\phi_{\mathrm{ave}}(t)$ as a function of time for several values of asymmetry, $1.518 \leq \alpha \leq 1.746$, for $T=0.22$. Not surprisingly, as $\alpha \rightarrow \alpha_{c}=1.5$, the field remains longer in the metastable state, since the nucleation energy barrier $E_{b} \rightarrow \infty$ at $\alpha_{c}$. However, a quick glance at the time axis shows the fast decay time-scale, of order $10^{1-2}$. For comparison, for $1.518 \leq$ $\alpha \leq 1.56$, HN would predict nucleation time-scales of order $\sim 10^{28} \geq \tau_{\mathrm{HN}} \sim \exp \left[E_{b} / T\right] \geq 10^{12}$ (in dimensionless units). [The related nucleation barriers with the effective potential are $E_{b}(\alpha=1.518)=14.10$ and $E_{b}(\alpha=1.56)=5.74$.] For small asymmetries $\phi_{\text {ave }}(t)$ displays similar oscillatory behavior to the SDW case before transitioning to the global minimum. As $\alpha$ is increased the number of oscillations decreases. For large asymmetries, $\alpha \geq 1.746$, the entire field crosses over to the global minimum without any nucleation event, resulting in oscillations about the global minimum. This is situation described in case i) above.

In Fig. 6 we show the ensemble-averaged nucleation timescales for resonant nucleation, $\tau_{\mathrm{RN}}$, as a function of the nucleation barrier (computed with eq. 2), $E_{b} / T$, for the temperatures $T=0.18,0.20$, and 0.22 . [For temperatures above $T=0.26$ we are in the vicinity of the critical point in which no barrier exists.] The nucleation time was measured when $\phi_{\text {ave }}$ crosses the maximum of $V_{\text {eff. }}$ The best fit is a power law:

$$
\tau_{R N} \propto\left(E_{b} / T\right)^{B}
$$

with $B=3.762 \pm 0.016$ for $T=0.18, B=3.074 \pm 0.015$ for $T=0.20$, and $B=2.637 \pm 0.018$ for $T=0.22$. This simple power law holds for the same range of temperatures where we have observed the synchronous emergence of oscillons. It is not surprising that the exponent $B$ increases with decreasing 


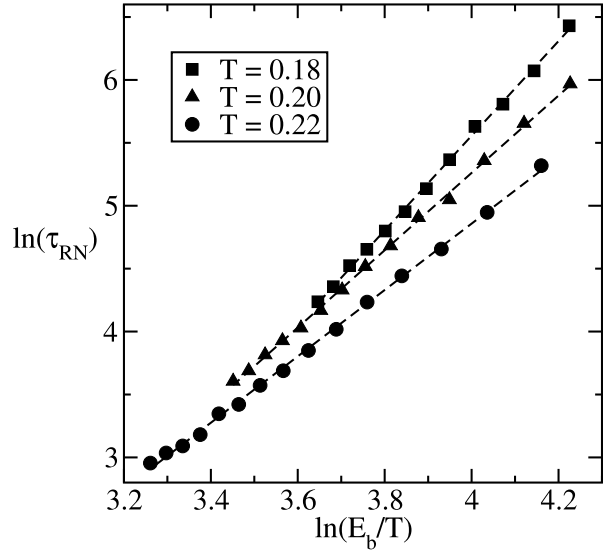

FIG. 6: Decay time-scale $\tau_{\mathrm{RN}}$ as a function of critical nucleation effective free-energy barrier $E_{b} / T$ at $T=0.18,0.20$, and $T=0.22$. The best fits (dashed lines) are power-laws with exponents $B \simeq 3.762$, 3.074 , and 2.637, respectively.

$T$, since the synchronous emergence of oscillons becomes less pronounced and eventually vanishes. In these cases we should expect a smooth transition into the exponential time-scales of HN.

We conclude that fast quenching can dramatically affect the nucleation time-scale of first order phase transitions. In other words, HN fails for fast quenches.

Here we propose the mechanism by which this fast decay occurs: for nearly degenerate potentials, $\alpha_{c}<\alpha \leq \alpha_{\mathrm{I}}$, the critical nucleus has a much larger radius than a typical oscillon; it will appear as two or more oscillons coalesce. We call this Region I, defined for $R_{b} \geq 2 R_{\mathrm{osc}}$, where $R_{\mathrm{osc}}$ is the minimum oscillon radius computed from Ref. [5]. Figure 7 illustrates this mechanism. Two oscillons, labeled A and B, join to become a critical nucleus. [The interested reader can see simulation movies at http://www.dartmouth.edu/ cosmos/oscillons.]

As $\alpha$ is increased further, the radius of the critical nucleus decreases, approaching that of an oscillon. In this case, a single oscillon grows unstable to become the critical nucleus promoting the fast decay of the metastable state: there is no coalescence. We call this Region II, $\alpha_{\mathrm{I}}<\alpha \leq \alpha_{\mathrm{II}}, R_{b}<2 R_{\mathrm{Osc}}$. This explains the small number of oscillations on $\phi_{\text {ave }}(t)$ as $\alpha$ is increased [cf. Fig. 5]. To corroborate our argument, in Fig. 8 we contrast the critical nucleation radius with that of oscillons as obtained in Ref. [5], for different values of effective energy barrier and related values of $\alpha$ at $T=0.22$. The critical nucleus radius $R_{b}$ is equal to $2 R_{\mathrm{osc}}$ for $\alpha=1.547$. This defines the boundary between Regions I and II: for $\alpha \geq \alpha_{\text {I }}$ a single oscillon may grow into a critical bubble. Finally, for $\alpha \geq \alpha_{\text {II }}=1.746$ the field crosses over to the global minimum without any nucleation event.

An obvious extension of the present work is the investigation of "resonant nucleation" in 3d. Preliminary results indicate that the power law behavior persists with $B \sim 1.5$ for the relevant range of temperatures for oscillon coalescence. In general, RN will occur whenever the effective potential changes faster than the typical relaxation rate of the longest wavelength of the order parameter. These results could be ex-
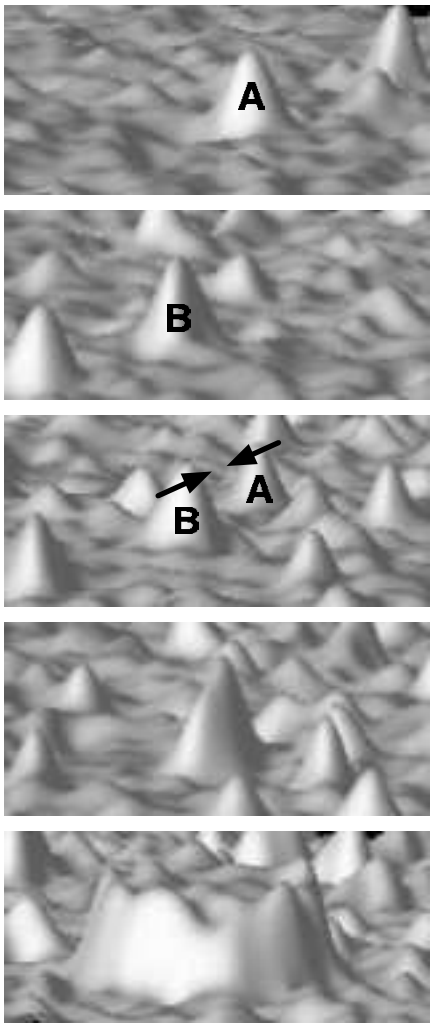

FIG. 7: Two oscillons coalesce to form a critical bubble. First two frames from top show oscillons A and B. Third and fourth frames shows A and B coalescing into a critical bubble. Final frame shows growth of bubble expanding into metastable state.

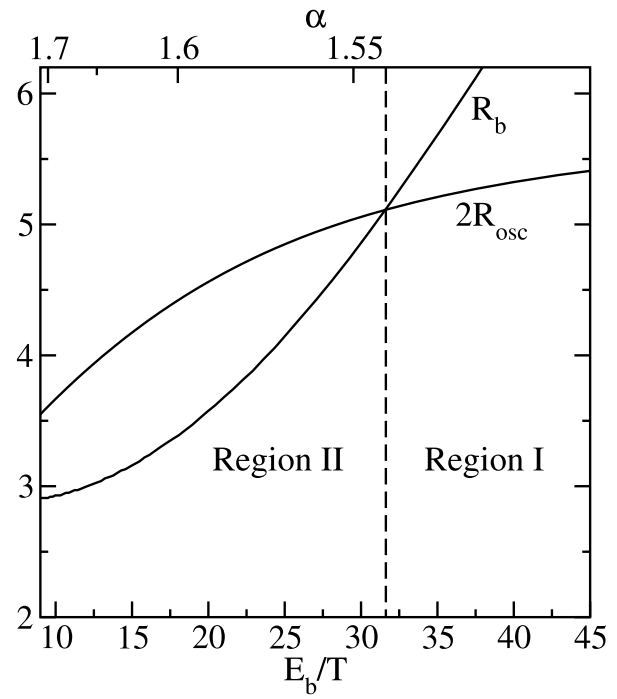

FIG. 8: Radius of critical bubble $\left(R_{b}\right)$ and twice the minimum oscillon radius $\left(2 R_{\text {osc }}\right)$ as a function of its energy barrier and related values of $\alpha$ at $T=0.22$. For $\alpha \geq 1.547$ one cannot easily distinguish between an oscillon and a critical bubble. 
tended to systems in the Ising universality class, in particular to ferromagnetic transitions. They could also be applied in cosmology, to where we turn to next.

\section{RESONANT INFLATION: CAN OLD INFLATION BE RESCUED?}

The simple elegance of the original "Old" Inflation (OI) scenario proposed by Guth in 1981 has, since then, inspired many variations $[15,16]$. More than just the elegance of its formulation, based on a single scalar field decaying from an initial metastable state to a lower-energy state by bubble nucleation, the original OI model had a clear connection with particle physics: the inflaton was to be the same scalar field promoting the symmetry breaking of Grand Unified models, linking early-Universe cosmology to high-energy particle physics. In fact, it is this particle physics connection that motivated and motivates the widespread use of scalar fields in early-Universe physics.

Unfortunately, Guth's original proposal didn't work. As he himself argued, and then Linde, and Albrecht and Steinhardt[17], the bubble-nucleation rate could not compete with the exponential expansion rate of the Universe: the transition would never end. Roughly, while bubble walls expanded with the speed of light, their centers receded from each other exponentially fast, making it impossible for the walls to touch, the bubbles to coalesce, and the transition to complete. Old Inflation gave rise to a universe with inhomogeneities incompatible with the observed smoothness of the cosmic microwave background[18]. Guth and Weinberg[19], and later Turner, Weinberg, and Widrow[20], performed a detailed analysis of the constraints needed to render OI and OIinspired scenarios viable. They concluded that a strong (or, equivalently, slow) first order phase transition based on a single scalar field could not be made to work: the ratio of decay rate to the expansion rate per unit volume $\left[H^{4} \simeq\left(T^{2} / M_{\mathrm{Pl}}\right)^{4}\right]$, had to be sufficiently small

$$
\varepsilon \equiv \Gamma / H^{4} \leq 10^{-4},
$$

initially, so that early bubbles didn't produce inhomogeneities during nucleosynthesis and on the CMB. (For $\Gamma \simeq$ $T^{4} \exp [-E(T) / T]$ and $T_{\mathrm{GUT}}=10^{15} \mathrm{GeV}$, this implies that $E(T) / T \gtrsim 46.1$ initially). On the other hand, it had also to grow by the end of inflation $(\varepsilon \rightarrow 9 \pi / 4)$ to guarantee that the transition was completed[20]. [This implies $E(T) / T \lesssim 34.9$.] In other words, successful inflation forced the decay rate to be time-dependent: small at the beginning of inflation and of order unity at the end. As further work has shown, this could be achieved by invoking more fields[21] and/or a nonminimal gravitational coupling[22].

Given what we have learned in the previous section about resonant nucleation, it is natural to wonder whether such effects can play a role on inflation. If we write $\varepsilon_{H N} \simeq$ $T^{4} \exp [-E(T) / T]$ to represent the ratio of eq. 6 using the homogeneous nucleation rate, and $\varepsilon_{R N} \simeq T^{4}[E(T) / T]^{-B}$ the ratio using the $\mathrm{RN}$ rate, equality is attained whenever

$$
B=\beta / \ln \beta \text {, }
$$

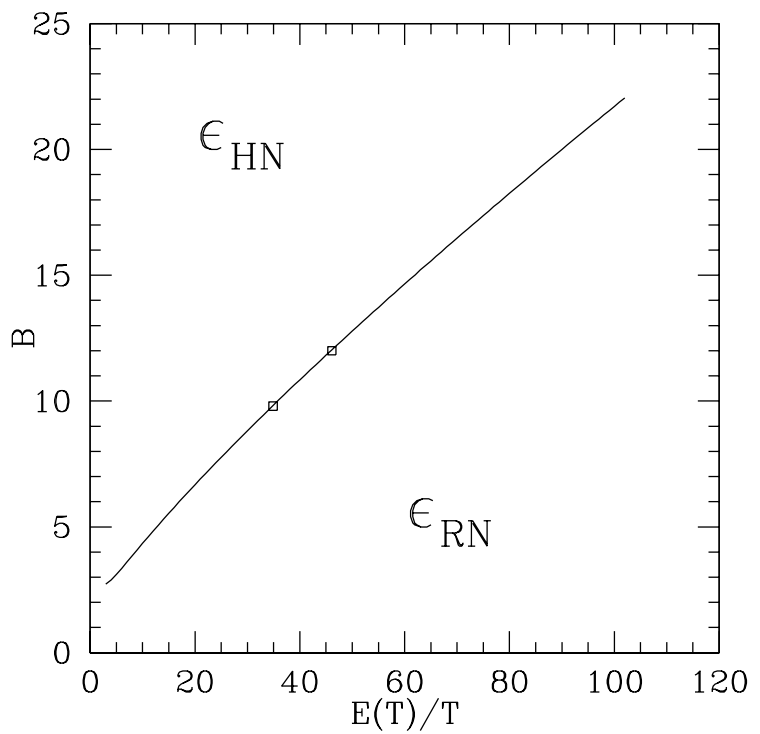

FIG. 9: Comparison of homogeneous nucleation (HN) and resonant nucleation (RN) with power $B$ in an expanding Universe. For a fixed B and nucleation barrier $\beta=E(T) / T$ (or $S_{b}$ at $T=0$ ), the line denotes equality. Values above the curve imply faster $\mathrm{HN}$, while those below imply faster RN.

where $\beta \equiv E(T) / T$ (or $\equiv S_{b}$ at $T=0$ ). In Fig. 9, $B$ is shown for representative values of the nucleation barrier $\beta$. The line denotes $\varepsilon_{\mathrm{HN}} / \varepsilon_{\mathrm{RN}}=1$. The squares denote the limits imposed by the inflationary constraints of Ref. [20]. For these values of $\beta$, unless $B \gtrsim 9$, which is very unlikely, resonant nucleation rates are always faster. In $d=2$, where $B \simeq 3$, it is clear that $\varepsilon_{H N} / \varepsilon_{R N}<1$ for all realistic values of $\beta$, not a surprising result.

Why is this useful for inflation? For successful inflation with HN, the constraints of Ref. [20] limit the nucleation barrier $\beta$ to be fairly small $\mathrm{O}(\sim 40)$. [See Fig. 9.] However, calculations of bounce actions show that $\beta$ usually scales with inverse powers of coupling constants. These two requirements compete with each other, making it hard to have small nucleation barriers with small couplings. Applying the percolation constraint to the RN rate, one obtains $\beta^{B} \sim 10^{16}$. For $B=3$, this gives $\beta \sim 10^{16 / 3}$ : small couplings (or, equivalently, large barriers) are easier to accommodate with RN, the first reason why it may be useful for inflation.

The second and most important reason is that $\mathrm{RN}$ makes it much easier to complete the transition. Clearly, if some mechanism capable of producing the same net effect as the fast quenching responsible for $\mathrm{RN}$ was present in the early Universe, an initially slow first order transition could become fast at some point, going from an exponential to a power law decay. In this way, even a potential with a large initial barrier would not be an impediment to the successful termination of inflation. One possible way of implementing $\mathrm{RN}$ in cosmology is to invoke a second field $\psi$ that couples to the nucleating field $\phi$ in a way somewhat reminiscent of hybrid inflation. In that model the inflaton $\phi$ is coupled quadratically to another scalar field $\psi$ which has a symmetric double well 
potential[21]:

$$
V(\phi, \psi)=\frac{1}{4 \lambda}\left(M^{2}-\lambda \psi^{2}\right)^{2}+\frac{m^{2}}{2} \phi^{2}+\frac{g^{2}}{2} \phi^{2} \psi^{2} .
$$

Inflation is driven by the energy density $V(\phi, 0)$ while the inflaton $(\phi)$ is rolling down along the $\psi=0$ valley[16]. As $\phi$ reaches a critical value, $\psi$ becomes spinodally unstable and quickly rolls to one of the minima (or both, but this creates domain walls, another problem), terminating inflation abruptly.

The key difference with the mechanism being proposed here is that bubble nucleation still occurs at the end of inflation. A possible name is hence resonant inflation (RI): it blends OI with the physics of resonant nucleation.

Modify the potential for the field $\phi$ that gives rise to $\mathrm{RN}$ by coupling another field $(\psi)$ quadratically to it as follows,

$$
\begin{aligned}
V(\phi, \psi)= & \frac{1}{2}\left(m^{2}+g^{2} \psi^{2}\right) \phi^{2}-\frac{\alpha}{3} \phi^{3}+\frac{\lambda}{8} \phi^{4}+ \\
& +\frac{1}{2} m_{\psi}^{2} \psi^{2}+\left|V\left(\phi_{+}, 0\right)\right|
\end{aligned}
$$

where $\phi_{+}$is the value of $\phi$ at the global minimum of $V(\phi, 0)$ so that $V(0, \psi)$ provides the net vacuum energy responsible for inflation. [Note that here the inflaton is $\psi$.] Inflation lasts while $\psi$ is rolling down the $\phi=0$ valley. Notice that the mass term for $\phi, M_{\phi}^{2}=m^{2}+g^{2} \psi^{2}$, decreases as $\psi$ rolls down its potential. While $M_{\phi}^{2}>\alpha^{2} / 2 \lambda$, the only minimum in the $\phi$ direction is at $\phi=0$. However, as $\psi$ decreases, $M_{\phi}^{2}$ will eventually drop below $\alpha^{2} / 2 \lambda$ and a new minimum will appear at $\phi_{+}=\frac{\alpha}{\lambda}\left[1+\left(1-2 M_{\phi}^{2} \lambda / \alpha^{2}\right)^{1 / 2}\right]$. At $M_{\phi}^{2}=\frac{4 \alpha^{2}}{9 \lambda}$, the two minima are degenerate. At this point, as $\psi$ continues to approach zero, the minimum at $\phi=0$ becomes metastable. Oscillations in $\phi$, induced by the decrease in its mass, will induce RN. This will be true as long as the decrease in $M_{\phi}, \dot{M}_{\phi} \simeq \frac{g^{2}}{m} \psi \dot{\psi}$, is fast enough. [It was assumed for simplicity that $g^{2} \psi^{2} / m^{2} \ll 1$ which is not true for very small $\psi$.]

For RI to work, $\dot{M}_{\phi} / M_{\phi}<H$ during inflation and $\dot{M}_{\phi} / M_{\phi}>$ $H$ after it. During inflation, with a slow-roll approximation, $\psi \dot{\psi} \simeq-\frac{m_{\psi}^{2}}{3 H} \psi^{2}$. We then obtain,

$$
\dot{M}_{\phi} \simeq-g^{2} \frac{m_{\psi}^{2}}{3 H m} \psi^{2}
$$

Also, if $N$ is the number of $e$-folds, $\psi_{e}^{2}=\psi_{i}^{2}-\frac{M_{P l}^{2}}{2 \pi} N$, where $\psi_{i(e)}$ is the value of the field $\psi$ at the beginning (end) of the inflationary period. [For simplicity, it was assumed that during inflation $\frac{1}{2} m_{\psi}^{2} \psi^{2}>\left|V\left(\phi_{+}, 0\right)\right|$, that is, inflation is dominated initially by the vacuum energy of the inflaton field $\psi$.] Slow-roll ends when $\psi_{e}^{2} \lesssim M_{P l}^{2} / 12 \pi$. Using this result and eq. 10 , the slow variation of $M_{\phi}$ implies, $g^{2}(12 \pi)^{1 / 2}<\left(m / M_{P l}\right)^{2}$. [For $m \sim 10^{16} \mathrm{GeV}, g<4 \times 10^{-4}$.] This condition is also consistent with the approximation $g^{2} \psi^{2} / m^{2} \ll 1$ for $\psi_{i}>\psi>\psi_{e}$, that is, during inflation.

If slow-roll ends when the minimum in $\phi_{+}$appears, we obtain (this is similar to the critical condition in hybrid inflation[21]),

$$
\frac{\alpha_{0}^{2}}{2 \lambda}=1+\frac{g^{2} M_{P l}^{2}}{12 \pi m^{2}}
$$

where we defined for convenience $\alpha \equiv m \alpha_{0}$. The condition for slow variation of $M_{\phi}$ during inflation forces the second term on the rhs of eq. 11 to be very small. Thus, if we want to impose that the $\phi_{+}$minimum appears close to the end of inflation, we must have $\alpha_{0}^{2} / 2 \lambda \sim 1$, not a difficult condition to satisfy.

As inflation ends, $\psi$ will start rolling down fast towards the $\psi=0$ minimum and oscillate around it. Since in this regime, $\dot{M}_{\phi} / M_{\phi} \sim\left(g^{2} / m^{2}\right) \dot{\psi} \psi$, the rapid motion of $\psi$ will induce the time-dependence in $M_{\phi}$ needed to trigger resonant bubble nucleation. In order for the transition to end successfully, the percolation constraint $\varepsilon_{\mathrm{RN}}>9 / 4 \pi$, must be satisfied. This implies,

$$
\left(S_{b}\right)^{B}<\frac{4 \pi}{9}\left(\frac{M_{P l}}{m}\right)^{4} .
$$

If $m \sim 10^{16} \mathrm{GeV}$ and $B \sim 2$ (as indicated by preliminary results in $d=3$ ), RI terminates efficiently if $S_{b} \lesssim 10^{6}$. Since the inflationary phase is due to the slow-roll dynamics of the $\psi$ field and not by the metastable field $\phi$, the percolation constraint can be satisfied by a wide range of couplings. Also, since $\phi=0$ only becomes metastable after the end of slow roll, there is no need to impose the big bubble constraint: once $\psi$ starts rolling fast at the end of inflation, $\mathrm{RN}$ will ensue and rapid bubble nucleation and coalescence will quickly reheat the Universe. Although several details remain to be worked out, this preliminary analysis indicates that resonant nucleation can be successfully applied to inflationary cosmology.

The author would like to thank the organizers for their kind hospitality.
[1] A. Einstein, Ann. Phys. 17, 549 (1905).

[2] P. Hänggi and F. Marchesoni, "100 Years of Brownian Motion," submitted to Chaos, [cond-mat/0502053].

[3] D. Walgraef, Spatio-Temporal Pattern Formation Springer, New York, 1997; M. C. Cross and P. C. Hohenberg, Rev. Mod. Phys. 65, 851 (1993).

[4] A. Vilenkin and E. P. S. Shellard, Cosmic Strings and Other
Topological Defects, Cambridge University Press, Cambridge, 1994.

[5] M. Gleiser, Phys. Lett. B 600, 126 (2004).

[6] M. Gleiser, Int. J. Mod. Phys. D, in press [hep-th/0602187].

[7] G. Aarts, G. F. Bonini, and C. Wetterich, Phys. Rev. D 63, 025012 (2000); G. Aarts, G. F. Bonini, and C. Wetterich, Nucl. Phys. B 587, 403 (2000). 
[8] J. D. Gunton, M. San Miguel, and P. S. Sahni, in Phase Transitions and Critical Phenomena, edited by C. Domb and J. L. Lebowitz, v. 8, Academic Press, London, 1983; J. D. Gunton, J. Stat. Phys. 95, 903 (1999); J. S. Langer, in Solids Far from Equilibrium, edited by C. Godrèche, Cambridge University Press, Cambridge, 1992.

[9] D. K. Campbell, J. F. Schonfeld, and C. A. Wingate, Physica 9D, 1 (1983); R. Rajamaran, Solitons and Instantons, NorthHolland, Amsterdam, 1987.

[10] M. Gleiser, Phys. Rev. D 49, 2978 (1994); E. J. Copeland, M. Gleiser, and H. R. Muller, Phys. Rev. D 52, 1920 (1995); E. B. Bogomol'nyi, Sov. J. Nucl. Phys. 24, 449 (1976); M. Gleiser and A. Sornborger, Phys. Rev. E 62, 1368 (2000); A. Adib, M. Gleiser, and C. Almeida, Phys. Rev. D 66, 085011 (2002).

[11] M. Gleiser and R. Howell, Phys. Rev. E 68, 065203(R) (2003).

[12] M. Gleiser and R. Howell, Phys. Rev. Lett. 94, 151601 (2005).

[13] S. Coleman, Phys. Rev. D 15, 2929 (1977); C. Callan and S. Coleman, Phys. Rev. D 16, 1762 (1977); A. Linde, Nucl. Phys. B 216, 421 (1983); [Erratum: B 223, 544 (1983)]
[14] M. Gleiser, "Oscillons in Scalar Field Theories: Applications in Higher Dimensions and Inflation," in press Int. J. Mod. Phys. D, [hep-th/0602187].

[15] A. H. Guth, Phys. Rev. D 23, 347 (1981).

[16] For a recent review see, B. A. Bassett, S. Tsujikawa, and D. Wands, astro-ph/0507632, to appear in Rev. Mod. Phys.

[17] A. D. Linde, Phys. Lett. B 108, 389 (1982); A. Albrecht and P. Steinhardt, Phys. Rev. Lett. 48, 1220 (1982).

[18] H. V. Peiris et al., Astrophys. J. Suppl. 148, 213 (2003); C. L. Bennet et al., ibid. 148, 1 (2003).

[19] A. H. Guth and E. J. Weinberg, Nucl. Phys. B 212, 321 (1983).

[20] M. S. Turner, E. J. Weinberg, Phys. Rev. D 46, 2384 (1992).

[21] A. D. Linde, Phys. Rev. D 49, 748 (1994).

[22] A. D. Dolgov, in The Very Early Universe, ed. by G. W. Gibbons, S. W. Hawking, and S. T. C. Siklos (Cambridge University Press, Cambridge, 1983). For a recent work see, F. di Marco and A. Notari, astro-ph/0511396. 